\title{
Flow-limiting thrombosis after intracoronary coil embolisation: optical coherence tomography during acute myocardial infarction
}

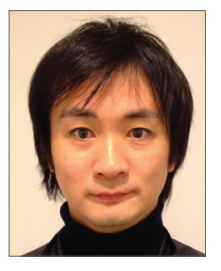

Nobuaki Kobayashi ${ }^{1 *}$, MD, PhD; Masamichi Takano², MD, PhD; Tetsuro Shimura ${ }^{2}, \mathrm{MD}$;

Noritake Hata ${ }^{1}, \mathrm{MD}, \mathrm{PhD}$; Wataru Shimizu ${ }^{3}, \mathrm{MD}, \mathrm{PhD}$

1. Division of Intensive Care Unit, Chiba Hokusoh Hospital, Nippon Medical School, Chiba, Japan;

2. Department of Cardiology, Chiba Hokusoh Hospital, Nippon Medical School, Chiba, Japan; 3. Department of Cardiovascular Medicine, Nippon Medical School, Tokyo, Japan

This paper also includes supplementary data published online at: http://www.pcronline.com/eurointervention/92nd_issue/209

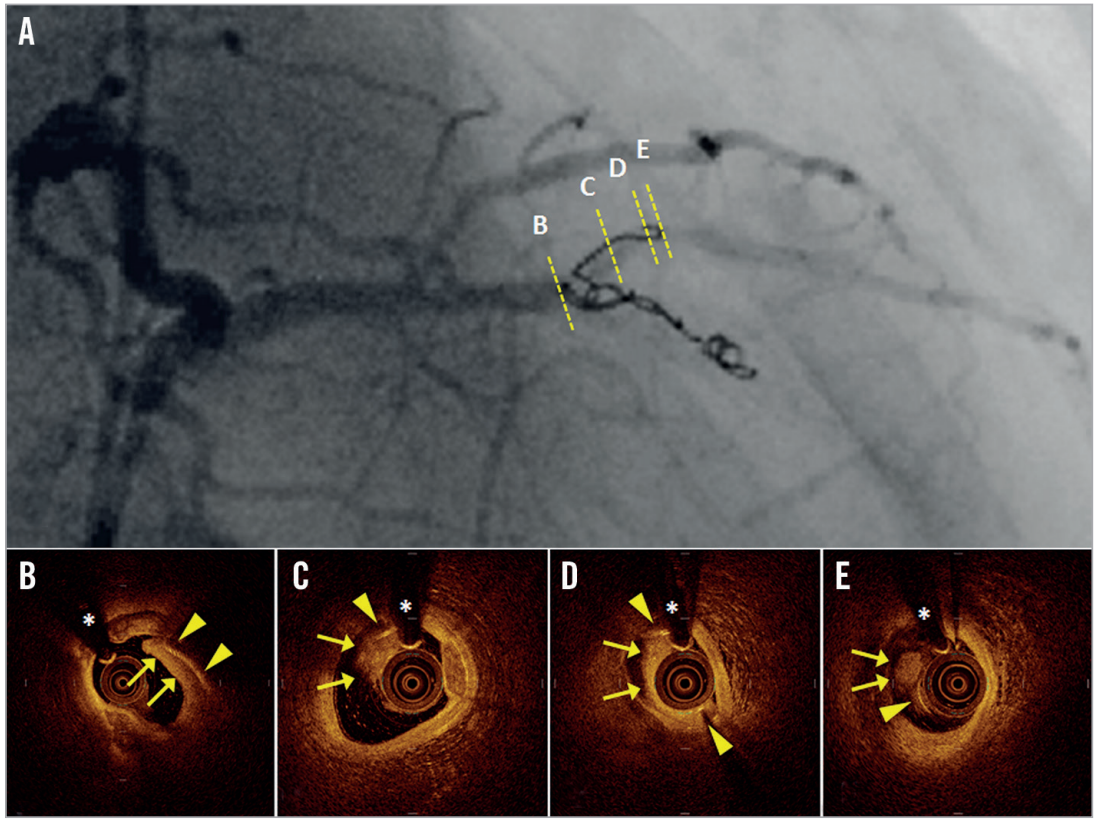

A 75-year-old female was transferred to our hospital with sudden onset chest pain radiating to the left arm. She had a history of hypertension, chronic kidney disease treated with regular haemodialysis, and a prior anterior myocardial infarction. Electrocardiography upon admission showed ST-segment elevation in broad anterior leads. Previous coronary angiography had illustrated chronic total occlusion (CTO) at the midleft anterior descending artery (LAD) and collateral flow from the 2nd diagonal branch (DB) to the distal LAD through the 3rd DB. Percutaneous coronary intervention for the CTO lesion had been performed at another hospital five days prior to admission. During this procedure, coil embolisation with the Tornado ${ }^{\circledR}$ Embolization Coil (Cook Japan Incorporated, Tokyo, Japan) had been performed to treat the complication of LAD perforation.

During urgent coronary catheterisation, part of the embolisation coil was found to have migrated from the LAD to the 2nd DB, and delayed flow of the 2nd DB was observed (panel A, Moving image 1). Optimal coherence tomography (OCT) showed massive thrombi (arrows) surrounding the embolisation coil (arrowheads; asterisks indicate guidewire artefacts) (panel B-panel E, Moving image 2). A drug-eluting stent was then implanted in order to attach the floating coil and thrombi to the vessel wall, with angiographic flow improving following the stenting (Online Figure 1A, Moving image 3). Although stent deployment brought about clear lumen dilatation, the embolisation coil between the stent struts partially protruded into the lumen (Online Figure 1B-Online Figure 1E, Moving image 4). According to our OCT images, the Tornado coil partially migrated to the 2nd DB, inviting formation of flow-limiting thrombi, which was the mechanism of the acute myocardial infarction.

\section{Conflict of interest statement}

The authors have no conflicts of interest to declare.

\section{Supplementary data}

The legends of Online Figure 1 and the Moving images can be found in the online version of the paper.

*Corresponding author: Division of Intensive Care Unit, Chiba Hokusoh Hospital, Nippon Medical School, 1715 Kamagari, Inzai, Chiba 270-1694, Japan.E-mail: s5047@nms.ac.jp 


\section{Supplementary data}

Moving image 1. Initial coronary angiography showed delayed coronary flow related to an embolisation coil that had migrated from the left anterior descending artery to the 2nd diagonal branch. Moving image 2. Initial optical coherence tomography illustrated luminal narrowing due to massive thrombi surrounding the coil.
Moving image 3. Coronary angiography after stent deployment showed improved coronary flow.

Moving image 4. Optical coherence tomography after stent deployment showed clear lumen dilatation. The embolisation coil was visible behind the stent struts and partially protruded into the lumen.
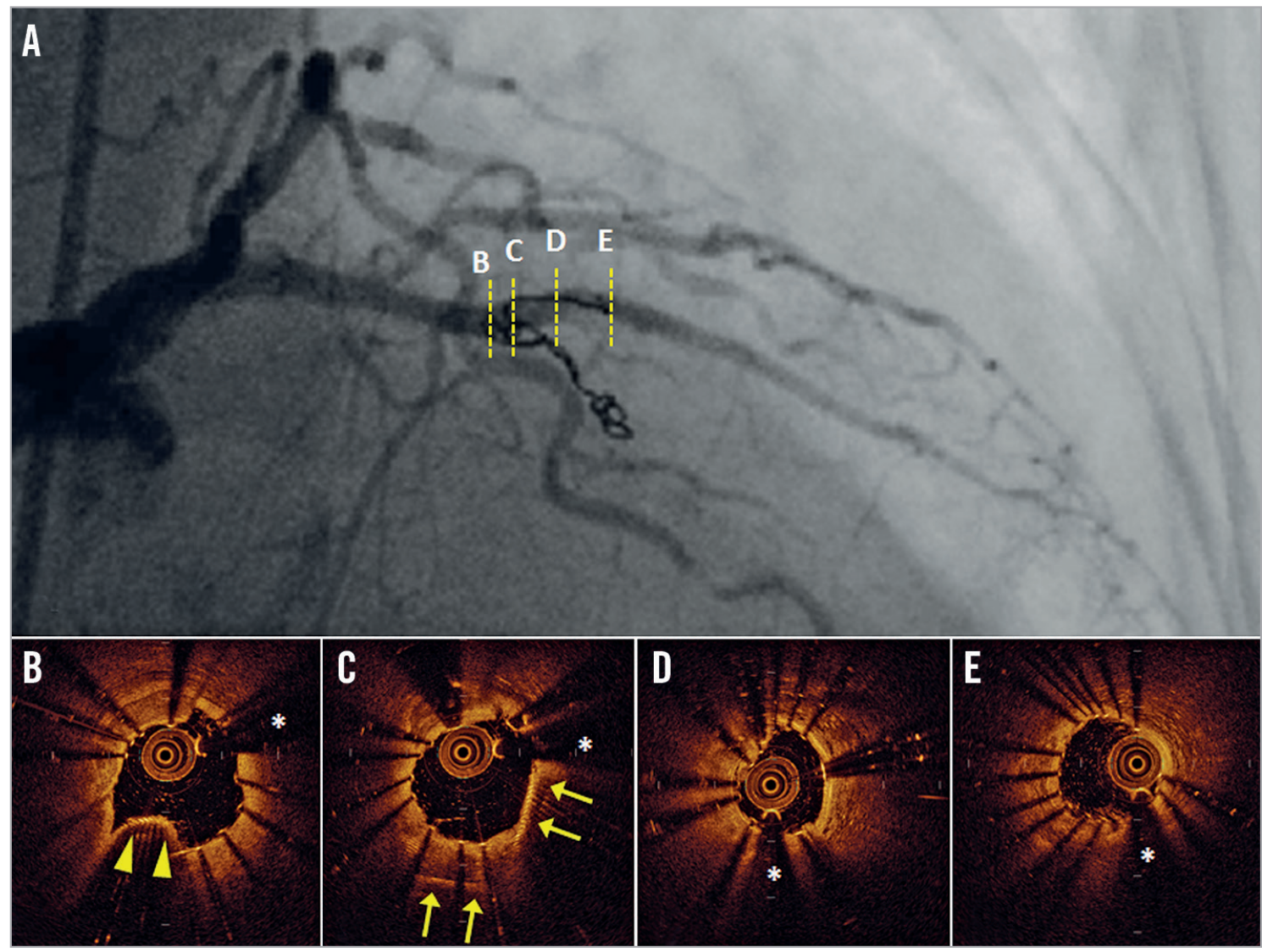

Online Figure 1. Images after stent implantation. Coronary angiography after stent implantation (A) showed improved coronary flow. Optical coherence tomography images after stent deployment $(B-E)$ showed the stent confining the embolisation coil behind it (arrows), although part of the coil protruded beyond the stent struts (arrowheads). Asterisks indicate guidewire artefacts. 